International Research Journal of Engineering Science, Technology and Innovation (IRJESTI) (ISSN2315-5663) Vol. 3(4) pp. 35-42, September 2014

DOI: http:/dx.doi.org/10.14303/irjesti.2014.068

Available online http://www.interesjournals.org/IRJESTI

Copyright @ 2014 International Research Journals

Review

\title{
Feynman propagator, ermakov-lewis invariant and bohmian trajectories for the logarithmic nonlinear schrödinger-nassar equation
}

\author{
"José Maria Filardo Bassalo, ${ }^{2}$ Antonio Boulhosa Nassar and ${ }^{3}$ Mauro Sérgio Dorsa Cattani \\ ${ }^{1}$ Academia Paraense de Ciências, 66035-400, Belém,PA ${ }^{2}$ Extension Program-Department of Sciences, University of \\ California, Los Angeles, California $90024{ }^{3}$ Instituto de Física da USP, 05389-970, São Paulo, SP
}

*Corresponding authors e-mail: jmfbassalo@gmail.com

Abstract

\begin{abstract}
In this paper we study the Feynman Propagator, the Ermakov-Lewis invariant and the Bohmian Trajectories for the Logarithmic Nonlinear Schrödinger-Nassar Equation, linearized along a classical trajectory, by using the quantum mechanical formalism of the de Broglie-Bohm.
\end{abstract}

Keywords:De Broglie-Bohm Quantum Mechanics; Feynman Propagator, Ermakov-Lewis Invariant, Bohmian Trajectory; Logarithmic Nonlinear Schrödinger-Nassar Equation.

PACS 03.65 - Quantum Mechanics

\section{INTRODUCTION}

The de Broglie-Bohm Quantum Mechanics and the Quantum Bohmian Trajectory

In 1948,(Feynman,1948) R. P. Feynman formulated the following principle of minimum action for the quantum mechanics:

The transition amplitude between the states / $a>$ and / $b$ $>$ of a quantum-mechanical system is given by the sum of the elementary contributions, one for each trajectory passing by $/ a>$ at the time $t_{a}$ and by $/ b>$ at the time $t_{b}$.

Each one of these contributions have the same modulus, but its phase is the classical action $S_{c \ell}$ for each trajectory. This principle is represented by the following expression known as the "Feynman propagator":

Error! , (1.1)

where $S(b, a)$ is the classical action given by:

$S(b, a)=\int_{t_{a}}^{t_{b}} L(x, \dot{x}, t) d t$,

$L(x, \dot{x}, t)$ is the Lagrangean and $D x(t)$ is the Feynman's Measurement. It indicates that we must perform the integration taking into account all the ways connecting the states $\mid a>$ and $\mid b>$.

The eq. (1.1) which defines $K(b, a)$ is called path integral or Feynman integral and the Schrödinger wavefunction $\Psi(x, t)$ of any physical system is given by (we indicate the initial position and initial time by $x_{o}$ and $t_{o}$, respectively):(

Feynman and Hibbs, 1965)

Error! , (1.3)

with the quantum causality condition:( Bernstein,1985)

Error! . (1.4)

The Logarithmic Nonlinear Schrödinger-Nassar Equation

In 2013,(Nassar,2013) A. Nassar proposed a logarithmic nonlinear Schrödinger equation to represent time dependent physical systems. In this article, let us considerer this same equation with a potential energy $V(x, t)$. Then, we have:

Error!

Error! , 
36 Int. Res. J. Eng. Sci. Technol. Innov.

where $\Psi(x, t)$ is a wave function which describes a given system and $K$ caracterizes the resolution of the measurement. The last term in the eq. (2.1) arises from the requirement that the integration of this equation with respect to the variable $x$ under the condition that for a particle the expectation value of the energy $<E(t)>$ defined as:

Error!, (2.2)

must be equal to the expectation values of the kinetic and potential energies.

\section{The Wave Packet of the Logarithmic Nonlinear} Schrödinger-Nassar Equation

Writting the wave function $\Psi(x, t)$ in the polar form defined by the Madelung-Bohm transformation:( Madelung, 1926, Bohm, 1952)

$\Psi(x, t)=\varphi(x, t) \times \exp [i S(x, t)], \quad(2.1 .1 .1)$

where $\varphi(x, t)$ will be defined in what follows.

Calculating the derivatives temporal and spatial of (2.1.1.1), we get:(Bassalo et al.,(2002)

Error! , (2.1.1.2a)

Error! . (2.1.1.2b)

Now, substituting the eqs. (2.1.1.2a,b) into the eq. (2.1) and remembering that exp $[i S]$ is common factor, we have:

Error!

Error!

$+\left(V(x, t)-i \hbar \mathrm{\kappa}\left[\ln (\varphi)^{2}-<\ln (\varphi)^{2}>\right]\right) \varphi$

Multiplying the eq. (2.1.1.3) by Error! and separating the real and imaginary parts, results:

Error!, (2.1.1.4)

b) real part

Error! . (2.1.1.5)

\section{Dynamics of the Logarithmic Nonlinear Schrödinger- Nassar Equation}

Now, let us see the correlation between the expressions (2.1.1.4-5) and the traditional equations of the Ideal Fluid Dynamics (See books on the Fluid Mechanics, for instance: Streeter and Debler,1966, Coimbra,1967, Landau and Lifshitz 1969, Bassalo 1973, Cattani 1990/2005) a) Continuity Equation, b) Euler's equation. To do this let us perform the following correspondences:

Quantum density probability:

$$
|\Psi(x, t)|^{2}=\Psi^{*}(x, t) \Psi(x, t) \quad \leftrightarrow
$$

Quantum mass density: $\quad \rho(x, t)=\varphi^{2}(x, t) \quad \leftrightarrow$ $\sqrt{\rho(x, t)}=\varphi(x, t), \quad(2.1 .2 .1 \mathrm{a}, \mathrm{b})$

Gradient of the wave function: $\quad \frac{\hbar}{m} \frac{\partial S(x, t)}{\partial x} \leftrightarrow$
Quantum velocity: $\quad v_{q u}(x, t) \equiv v_{q u}, \quad(2.1 .2 .1 \mathrm{c}, \mathrm{d})$

Bohm quantum potential:

$V_{q u}(x, t) \equiv V_{q u}=-\frac{\hbar^{2}}{2 m} \frac{1}{\varphi} \frac{\partial^{2} \varphi}{\partial x^{2}}=-\frac{\hbar^{2}}{2 m} \frac{1}{\sqrt{\rho}} \frac{\partial^{2} \sqrt{\rho}}{\partial x^{2}}$

(2.1.2.1 e,f)

Putting the relations $(2.1 .2 .1 \mathrm{a}-\mathrm{d})$ into the equation (2.1.1.4) and considering that $\partial(\ln x) / \partial y=(1 / x)(\partial x / \partial y)$ and $\ln \left(x^{m}\right)=m \ln x$, we get:

\section{Error!}

Error!

$$
\begin{aligned}
& \frac{\partial(\ln \sqrt{\rho})}{\partial t}=-\frac{\hbar}{m} \frac{\partial S}{\partial x} \frac{\partial(\ln \sqrt{\rho})}{\partial x}-\frac{1}{2} \frac{\partial}{\partial x}\left(\frac{\hbar}{m} \frac{\partial S}{\partial x}\right)-\kappa[\ln (\rho)-<\ln (\rho)> \\
& \overrightarrow{\frac{1}{2}} \frac{1}{\rho} \frac{\partial \rho}{\partial t}=-v_{q u} \frac{1}{2} \frac{1}{\rho} \frac{\partial \rho}{\partial x}-\frac{1}{2} \frac{\partial v_{q u}}{\partial x}-\kappa[\ln (\rho)-<\ln (\rho)>] \\
& \rightarrow \\
& \frac{\partial \rho}{\partial t}=-v_{q u} \frac{\partial \rho}{\partial x}-\rho \frac{\partial v_{q u}}{\partial x}-2 \kappa[\ln (\rho)-<\ln (\rho)>] \rho \\
& \frac{\partial \rho}{\partial t}+\frac{\partial\left(\rho v_{q u}\right)}{\partial x}-2 \kappa[\ln (\rho)-<\ln (\rho)>] \rho
\end{aligned}
$$

expression that indicates decoherence of the physical system represented by the Logarithmic Nonlinear Schrödinger-Nassar Equation (LNLS-NE) [eq. (2.1)]; then the Continuity Equation its not preserved.

Now, let us obtained another dynamic equation of the LNLS-NE. So, differentiating the eq. (2.1.1.5) with respect $x$ and using the eqs. (2.1.2.1a-e), we obtain:

\section{Error!}

Error!

Error!

Error!

Error! . (2.1.2.3)

We must observe that the eq. (2.1.2.3) is an equation similar to the Euler Equation which governs the motion of a fluid particle.Considering the substantive differentiation (local plus convective) or hydrodynamic differentiation: (See books on the Fluid Mechanics, for instance)

\section{Error!, (2.1.2.4a)}

and that:

Error! , (2.1.2.4b)

the eq. (2.1.2.3) could be written as:

Error!

Error! (2.1.2.5)

We note that the eq. (2.1.2.5) has a form of the Second Newton Law, being the first term of the second member is the classical newtonian force and the second is the quantum bohmnian force. 


\section{The Quantum Wave Packet for the Logarithmic Nonlinear Schrödinger-Nassar Equation Linearized along a Classical Trajetory}

In order to find the quantum wave packet for the Logarithmic Nonlinear Schrödinger-Nassar Equation ( $L N L S-N E$ ) linearized along a classical trajetory, let us the considerer the ansatz: (Nassar,2013)

$$
\rho(x, t)=\left[2 \pi \delta^{2}(t)\right]^{-1 / 2} \times \exp \left\{-\frac{[x-q(t)]^{2}}{2 \delta^{2}(t)}\right\},
$$

(2.1.3.1a)

or [use the eq. (2.1.2.1a,b)]:

$$
\varphi(x, t)=\left[2 \pi \delta^{2}(t)\right]^{-1 / 4} \times \exp \left\{-\frac{[x-q(t)]^{2}}{4 \delta^{2}(t)}\right\},
$$

where $\delta(t)$ and $q(t)=\langle x\rangle$ are auxiliary functions of time, to will be determined in what follows, representing the width and the center of mass of wave packet, respectively.

Taking the eq. (2.1.3.1a), let us calculated the expressions [remember that $\ln (a b)=\ln a+\ln b$ and $\left.\ln e^{\alpha}=\alpha\right]$ :

$$
\ln [\rho(x, t)]=\ln \left(\left[2 \pi \delta^{2}(t)\right]^{-1 / 2} \times \exp \left\{-\frac{[x-q(t)]^{2}}{2 \delta^{2}(t)}\right\}\right)
$$

$=\ln \left[2 \pi \delta^{2}(t)\right]^{-1 / 2}-\frac{[x-q(t)]^{2}}{2 \delta^{2}(t)}$.

$<\ln [\rho(x, t)]>=<\ln \left(\left[2 \pi \delta^{2}(t)\right]^{-1 / 2} \times \exp \left\{-\frac{[x-q(t)]^{2}}{2 \delta^{2}(t)}\right\}\right)>$

$=\ln \left[2 \pi \delta^{2}(t)\right]^{-1 / 2}-<\frac{[x-q(t)]^{2}}{2 \delta^{2}(t)}>$.

Considering that:

Error! , (2.1.3.4)

and:

$<f(x, t)>=\int_{-\infty}^{+\infty} \rho(x, t) f(x, t) d x=g(t)$,

we have: (Bassalo,2010)

$\ln [\rho(x, t)]-<\ln [\rho(x, t)]>=-\frac{\delta^{2}(t)}{2 \rho(x, t)} \frac{\partial^{2} \rho(x, t)}{\partial x^{2}}$

Insering the eq. (2.1.3.6) into eq. (2.1.2.2), results:

$$
\frac{\partial \rho}{\partial t}+\frac{\partial\left(\rho v_{q u}\right)}{\partial x}=-2 k \rho\left(-\frac{\delta^{2}}{2 \rho} \frac{\partial^{2} \rho}{\partial x^{2}}\right) \quad \rightarrow
$$

$\frac{\partial \rho}{\partial t}+\frac{\partial\left(\rho v_{q u}\right)}{\partial x}-\frac{\partial}{\partial x}\left(D \frac{\partial \rho}{\partial x}\right)=0$

where:

$D=\mathrm{\kappa} \delta^{2}$.

Defining: (Nassar, 1986)

$\vartheta_{q u}=v_{q u}-\frac{D}{\rho} \frac{\partial \rho}{\partial x}$

then the eq. (2.1.3.7a) will be the form:

$\frac{\partial \rho}{\partial t}+\frac{\partial(\rho \vartheta)}{\partial x}=0$

Differenting the eq. (2.1.3.1a) in the variables $t$ and $x$ [remembering that $x$ and $t$ are independent variables], results: (Bassalo,2010)

$\frac{\partial \rho}{\partial t}=\rho\left\{-\frac{\dot{\delta}(t)}{\delta(t)}+\frac{\dot{q}(t)}{\delta^{2}(t)}[x-q(t)]+\frac{\dot{a}(t)}{\delta^{3}(t)}[x-q(t)]^{2}\right\}$ (2.1.3.10)

$\frac{\partial \rho}{\partial x}=-\rho \frac{[x-q(t)]}{\delta^{2}(t)}$.

Now, substituting the eqs. $(2 \cdot 1 \cdot 3 \cdot 10,11)$ into (2.1.3.9) and integrating the result, we obtain: (Bassalo,2010)

$\vartheta_{q u}(x, t)=\frac{\dot{\delta}(t)}{\delta(t)}[x-q(t)]+\dot{q}(t)$.

Using the eqs. $(2 \cdot 1 \cdot 3 \cdot 7 \mathrm{~b}, 8,12)$, we have:

$v_{q u}(x, t)=\left[\frac{\dot{\delta}(t)}{\delta(t)}-k\right] \times[x-q(t)]+\dot{q}(t)$.

To obtain the quantum wave packet $[\Psi(x, t)]$ of the $L N L S-N E$ given by eq. (2.1), let us expand the functions $\mathrm{S}(\mathrm{x}, \mathrm{t})$ and $V_{q u}(\mathrm{x}, \mathrm{t})$ around of $q(t)$ up to second Taylor order. In this way, we have:

Error! (2.1.3.14)

Error! (2.1.3.15)

$V_{q u}(x, t)=V_{q u}[q(t), t]+V_{q u}[q(t), t] \times[x-q(t)]+$

$+\frac{V^{\prime \prime}[\mathrm{qu}(\mathrm{t})}{\mathrm{t}] ; 2} \times[\mathrm{x}-\mathrm{q}(\mathrm{t})]^{2}$,

where (') and " means, respectively, Error! and Error!.

Differentiating the eq. (2.1.3.14) in the variable $x$, multiplying the result by $\hbar / m$, using the eqs. $(2 \cdot 1.2 .1 \mathrm{c}, d)$ and (2.1.3.13), results:

\section{Error!}


$=v_{q u}(x, t)=\left[\frac{\dot{\delta}(t)}{\delta(t)}-k\right] \times[x-q(t)]+\dot{q}(t)$

$S^{\prime}[q(t), t]=\frac{m \dot{q}(t)}{\hbar}, \quad S^{\prime \prime}[q(t), t]=\frac{m}{\hbar}\left[\frac{\dot{q}(t)}{\hbar}-\kappa\right]$.

(2.1.3.17a,b)

Substituting the eq. (2.1.3.17a,b) into the eq. (2.1.3.14), we have:

$S(x, t)=S_{0}(t)+\frac{m \dot{q}(t)}{\hbar} \times[x-q(t)]+\frac{m}{2 \hbar}\left[\frac{\dot{\delta}(t)}{\delta(t)}-\kappa\right] \times[x-q(t)]^{2}$

, (2.1.3.18a)

where:

$S_{0}(t) \equiv S[q(t), t], \quad(2 \cdot 1 \cdot 3 \cdot 18 b)$

are the quantum action.

Differentiating the eq. (2.1.3.18a) in relation to the time $t$, we obtain (remembering that $\partial x / \partial t=0$ ):

$\frac{\partial S}{\partial t}=\dot{S}_{0}(t)+\frac{\partial}{\partial t}\left\{\frac{m \dot{q}(t)}{\hbar} \times[x-q(t)]\right\}+\frac{\partial}{\partial t}\left\{\frac{m}{2 \hbar}\left[\frac{\dot{\delta}(t)}{\delta(t)}-\kappa\right] \times[x-q(t)]^{2}\right\}$

$\rightarrow$

$\frac{\partial S}{\partial t}=\dot{S}_{0}(t)+\frac{m \ddot{q}(t)}{\hbar} \times[x-q(t)]-\frac{m \dot{q}^{2}(t)}{\hbar}+$

$+$

$\frac{m}{2 \hbar}\left[\frac{\ddot{\delta}(t)}{\delta(t)}-\frac{\dot{\delta}^{2}(t)}{\delta^{2}(t)}\right] \times[x-q(t)]^{2}-\frac{m \dot{q}(t)}{\hbar}\left[\frac{\dot{\delta}(t)}{\delta(t)}-k\right] \times[x-q(t)]$

(2.1.3.19)

Considering the eqs. (2.1.2.1a) and (2.1.3.1b), let us write $V_{q u}$ given by eq. (2.1.2.1e) in terms of potencies of $[x-q(t)]$. Before, we calculate the following derivations:

Error!

Error!

Error!

Error!

Error!

Error!

Error! (2.1.3.20)

Substituting the eq. (2.1.3.20) in the equation (2.1.2.1e), taking into account the eqs. (2.1.3.16), and considering the identity of polynomial, results:

Error!

Error!

Error!

Error! (2.1.3.21)

Using the eqs. (2.1.1.5) and (2.1.2.1c,e), we have:

Error!

Error! (2.1.3.22)

Inserting the eqs. $(2 \cdot 1 \cdot 3 \cdot 13,15,19,21)$ into eq. (2.1.3.22), we obtain:
$\hbar\left\{\dot{S}_{0}(t)+\frac{m \ddot{q}(t)}{\hbar} \times[x-q(t)]-\frac{m \dot{q}^{2}(t)}{\hbar}+\frac{m}{2 \hbar}\left[\frac{\ddot{\delta}(t)}{\delta(t)}-\frac{\dot{\delta}^{2}(t)}{\delta^{2}(t)}\right] \times[x-q(t)]^{2}\right.$

$\left.\frac{m \dot{q}(t)}{\hbar}\left[\frac{\dot{\delta}(t)}{\delta(t)}-\kappa\right] \times[x-q(t)]\right\}+\frac{m}{2}\left\{\left[\frac{\dot{\delta}(t)}{\delta(t)}-\kappa\right] \times[x-q(t)]+\dot{q}(t)\right\}^{2}$

$+$

$+$

$V[q(t), t]+V^{\prime}[q(t), t] \times[x-q(t)]+\frac{V^{\prime \prime}[q(t), t]}{2} \times[x-q(t)]^{2}$

Error! (2.1.3.23)

Since $(x-q)^{0}=1$, we can gather together the eq. (2.1.3.22) in potencies of the $(x-q)$, obtaining:

$\left\{\hbar \dot{S}_{0}(t)-\frac{1}{2} m \dot{q}^{2}(t)+V[q(t), t]+\frac{\hbar^{2}}{4 m \delta^{2}(t)}\right\} \times[x-q(t)]^{0}$

$+m \ddot{q}(t)+V^{\prime \prime}[q(t), t] \times[x-q(t)]^{1}+$

$+\left\{\frac{m}{2}\left[\frac{\ddot{\delta}(t)}{\delta(t)}-2 \kappa \frac{\dot{\delta}(t)}{\delta(t)}+\kappa^{2}\right]+\right.$

$\left.+\quad \frac{1}{2} V^{\prime \prime}[q(t), t]-\frac{\hbar^{2}}{8 m \delta^{4}(t)}\right\} \times[x-q(t)]^{2}=0$.

(2.1.3.24)

As the above relation is an identically null polynomium, the coefficients of the potencies must be all equal to zero, that is:

$\dot{S}_{0}(t)=\frac{1}{\hbar}\left\{\frac{1}{2} m \dot{q}^{2}(t)+V[q(t), t]+\frac{\hbar^{2}}{4 m \delta^{2}(t)}\right\}$,

(2.1.3.25)

$\ddot{\delta}(t)-2 \kappa \dot{\delta}(t)+\kappa^{2} \delta(t)+\frac{1}{m} V^{\prime \prime}[q(t), t]=\frac{\hbar^{2}}{4 m^{2} \delta^{3}(t)}$,

(2.1.3.26)

$\ddot{q}(t)+\frac{1}{m} V^{\prime}[q(t), t]=0$.

Assuming that the following initial conditions are obeyed:

$q(0)=x_{0}, \dot{q}(0)=v_{0}, \delta(0)=\delta_{0}, \dot{\delta}(0)=b_{0}$,

(2.1.3.28a-d)

and that [see eqs. $(2 \cdot 1 \cdot 2 \cdot 1 \mathrm{c}, \mathrm{d})$ and (2.1.3.17b)]:

Error! , (2.1.3.29)

the integration of the expression (2.1.3.25) will be given by:

$S_{0}(t)=\frac{1}{\hbar} \int_{0}^{t} d t^{\prime}\left\{\frac{1}{2} m \dot{q}^{2}\left(t^{\prime}\right)-V\left[q\left(t^{\prime}\right), t^{\prime}\right]-\frac{\hbar^{2}}{4 m \delta^{2}\left(t^{\prime}\right)}\right\}+\frac{m v_{0} x_{0}}{\hbar}$.

(2.1.3.30) 
Taking into account the expressions $(2.1 .3 .18 a, b)$ in the equation (2.1.3.30) results:

$$
\begin{aligned}
& S(x, t)=\frac{1}{\hbar} \int_{0}^{t} d t^{\prime}\left\{\frac{m \dot{q}^{2}\left(t^{\prime}\right)}{2}-V\left[q\left(t^{\prime}\right), t^{\prime}\right]-\frac{\hbar^{2}}{4 m \delta^{2}\left(t^{\prime}\right)}\right\}+ \\
& + \\
& \frac{m v_{0} x_{0}}{\hbar}+\frac{m \dot{q}(t)}{\hbar} \times[x-q(t)]+\frac{m}{2 \hbar}\left[\frac{\dot{\delta}(t)}{\delta(t)}-\kappa\right] \times[x-q(t)]^{2}
\end{aligned}
$$

This result obtained above permit us, finally, to obtain the wave packet for the LNLS-NE. Indeed, considering the eqs. (2.1.1.1), (2.1.3.1b) and (2.1.3.31), we get:

$\left.\Psi(x, t)=[2 \pi \delta(t)]^{1 / 4} \times \exp \left\{\frac{i m}{2 \hbar}\left[\frac{\dot{\delta}(t)}{\delta(t)}-\kappa\right]-\frac{1}{4 \delta^{2}(t)}\right) \times[x-q(t)]^{2}\right\} \times$

$\times \exp \left\{\frac{i m \dot{q}(t)}{\hbar} \times[x-q(t)]+\frac{i m v_{0} x_{0}}{\hbar}\right\} \times$

$\times \exp \left\{\frac{i}{\hbar} \int_{0}^{t} d t^{\prime}\left[\frac{m \dot{q}^{2}(t)}{2}-V\left[q\left(t^{\prime}\right), t^{\prime}\right]-\frac{\hbar^{2}}{4 m \delta^{2}\left(t^{\prime}\right)}\right]\right\}$.

(2.1.3.32)

\subsubsection{Calculation of the Feynman Propagator for the Logarithmic Nonlinear Schrödinger-Nassar Equation Linearized along a Classical Trajetory}

The looked for Feynman-de Broglie-Bohm propagator for the Logarithmic Nonlinear SchrödingerNassar Equation (LNLS-NE) linearized along a classical trajectory, will be calculated using the eqs. (1.3) and (2.1.3.24). However, in the eq. (1.3), we will put with no loss of generality, $t_{o}=0$. Thus: (Bassalo et al., 2002)

Error! . (2.1.4.1)

Initially let us define the normalized quantity:

$\Phi\left(v_{o}, x, t\right)=(2 \pi \delta 2 ; 0)^{1 / 4} \Psi\left(v_{o}, x, t\right)$,

which satisfies the following completeness relation: (Bernstein,1985)

Error! . (2.1.4.3)

Considering the eqs. (2.1.1.1), (2.1.2.1a,b) and (2.1.4.2,3), we get:

$$
\begin{aligned}
& \Psi^{*}(x, t) \times \Psi(x, t)=\varphi^{2}(x, t)=\rho(x, t), \\
& \Phi^{*}\left(v_{0}, x, t\right) \Psi\left(v_{0}, x, t\right)= \\
& = \\
& \quad\left(2 \pi \delta_{0}^{2}\right)^{1 / 4} \Psi^{*}\left(v_{0}, x, t\right) \Psi\left(v_{0}, x, t\right)=\left(2 \pi \delta_{0}^{2}\right)^{1 / 4} \rho\left(v_{0}, x, t\right) \\
& \quad \rightarrow \\
& \rho\left(v_{0}, x, t\right)=\left(2 \pi \delta_{0}^{2}\right)^{-1 / 4} \Phi^{*}\left(v_{0}, x, t\right) \Psi\left(v_{0}, x, t\right) .
\end{aligned}
$$

(2.1.4.5)
On the other side, substituting the eq. (2.1.4.5) into eq. (2.1.3.9), integrating the result and using the expressions (2.1.3.1a) and (2.1.4.2) results [remembering that Error!, $\Psi^{*} \Psi( \pm \infty) \rightarrow 0$, and the integration for parts]:

$\frac{\partial\left(\Phi^{*} \Psi\right)}{\partial t}+\frac{\partial\left(\Phi^{*} \Psi \vartheta_{q u}\right)}{\partial x}=0 \rightarrow$

$\frac{\partial}{\partial t} \int_{-\infty}^{+\infty}\left(\Phi^{*} \Psi\right) d x+\int_{-\infty}^{+\infty} \frac{\partial\left(\Phi \Psi \vartheta_{q u}\right)}{\partial x} d x=$

$=\quad \frac{\partial}{\partial t} \int_{-\infty}^{+\infty}\left(\Phi^{*} \Psi\right) d x+\left.\left(\Phi^{*} \Psi \vartheta_{q u}\right)\right|_{-\infty} ^{+\infty}=$ $\frac{\partial}{\partial t} \int_{-\infty}^{+\infty}\left(\Phi^{*} \Psi\right) d x+\left.\left(2 \pi \delta_{0}^{2}\right)^{1 / 4}\left(\Psi^{*} \Psi \vartheta_{q u}\right)\right|_{-\infty} ^{+\infty} \rightarrow$ $\frac{\partial}{\partial t} \int_{-\infty}^{+\infty}\left(\Phi^{*} \Psi\right) d x=0$.

The eq. (2.1.4.6) shows that the integration is time independent. Consequently:

Error! . (2.1.4.7)

Multiplying the eq. (2.1.4.7) by $\Phi\left(v_{o}, x, t\right)$ and integrating over $v_{o}$ and using the eq. (2.1.4.3), we will obtain [remembering that Error!]:

\section{Error!}

= Error!

Error!

$=\int_{-\infty}^{+\infty} \int_{-\infty}^{+\infty} d v_{0} d x_{0} \Phi\left(v_{0}, x, t\right) \Phi^{*}\left(v_{0}, x_{0}, 0\right) \Psi\left(x_{0}, 0\right) \rightarrow$

Error!

$\left.\times \Phi^{*}\left(v_{0}, x_{0}, 0\right)\right\} \Psi\left(x_{0}, 0\right) d x_{0}$.

Comparing the eqs. $(2.1 .4 .1,8)$, we have:

Error! . (2.1.4.9)

Substituting the eqs. (2.1.3.31) and (2.1.4.2) in the equation (2.1.4.9), we obtain the Feynman Propagator for the LNLS-NE linearized along a classical trajetory, that we were looking for, that is [remembering that Error!]:

Error!

$\times \exp \left\{\left(\frac{i m}{2 \hbar}\left[\frac{\dot{\delta}(t)}{\delta(t)}-\kappa\right]-\frac{1}{4 \delta^{2}(t)}\right) \times[x-q(t)]^{2}\right\} \times$

$\times \exp \left\{i \frac{m \dot{q}(t)}{\hbar} \times[x-q(t)]\right\} \times$

$\left.\times \exp \left\{\frac{i}{\hbar} \int_{0}^{t} d t^{\prime}\left[\frac{m \dot{q}^{2}\left(t^{\prime}\right)}{2}-V\left[q\left(t^{\prime}\right), t^{\prime}\right)\right]-\frac{\hbar^{2}}{4 m \delta^{2}\left(t^{\prime}\right)}\right]\right\}$,

(2.1.4.10)

where $q(t)$ and $\delta(t)$ are solutions of the differential equations given by the eqs. $(2 \cdot 1 \cdot 3 \cdot 25,26)$. 
40 Int. Res. J. Eng. Sci. Technol. Innov.

Finally, it is important to note that putting $\mathrm{K}=0$ and $\left.Y q\left(t^{\prime}\right), t^{\prime}\right]=0$ into eq. $(2 \cdot 1 \cdot 4 \cdot 10)$ and eqs. $(2 \cdot 1 \cdot 3 \cdot 25,26)$ we obtain the free Feynman propagator. (Feynman and Hibbs, 1965) (Bassalo et al., 2002)

\section{Ermakov-Lewis Invariants}

Many years ago, in 1967 (Lewis, 1967), H. R. Lewis has shown that there is a conserved quantity, that will be indicated by $l$, associated with the time dependent harmonic oscillator $(T D H O)$ with frequency $\omega(t)$, given by:

$\mathrm{I}=\frac{1}{2}(\dot{\alpha} q-\dot{q} \alpha)^{2}+\left(\frac{q}{\alpha}\right)^{2}$

where $q$ and $\alpha$ obey, respectively the equations:

$$
\ddot{q}+\omega^{2}(t) q=0, \quad \ddot{\alpha}+\omega^{2}(t) \alpha=\frac{1}{\alpha^{3}} .
$$

On the other hand, as the above expressions have also been obtained by V. P. Ermakov (Ermakov, 1880) in 1880, the invariants determination of time dependent physical systems is also known as the Ermakov-Lewis problem. So, considerable efforts have been devoted to solve this problem and its generalizations, in the last forty years, and in many works have been published on these subjects (Nassar, 1986a).

\section{The Ermakov-Lewis Invariants for the Logarithmic Nonlinear Schrödinger-Nassar Equation}

Now, let us investigate the existence (or not) of these invariants for the Logarithmic Nonlinear SchrödingerNassar Equation (LNLS-NE) with the potential $V(x, t)$ given by:

Error!, (3.1.1)

which is the Time Dependent Harmonic Oscillator Potential (TDHOP).

Taking the eq. (2.1.2.3) and considering the eq. (3.1.1), results:

Error! . (3.1.2)

In order to integrate the eq. (3.1.2) let us assume that the expected value of quantum force [Error!] is equal to zero for all times $t$, that is:

$<\frac{\partial V_{q u}(x, t)}{\partial x}>\left.\rightarrow 0 \leftrightarrow \frac{\partial V_{q u}}{\partial x}\right|_{\mathrm{x}=\mathrm{q}(\mathrm{t})}, \quad<\mathrm{x}>=\mathrm{q}(\mathrm{t})$.

(3.1.3a-c)

In this way, using the eq. (2.1.2.1f), we can write the eq. (3.1.2) into two parts:

$\frac{\partial v_{q u}(x, t)}{\partial t}+v_{q u}(x, t) \frac{\partial v_{q u}(x, t)}{\partial x}+\omega^{2}(t) x=k(t) \times[x-q(t)]$ $(3.1 .4)$ $\frac{\partial}{\partial x}\left[\frac{\hbar^{2}}{2 m^{2}} \frac{1}{\sqrt{\rho(x, t)}} \frac{\partial^{2} \sqrt{\rho(x, t)}}{\partial x^{2}}\right]=k(t) \times[x-q(t)]$.

(3.1.5)

Performing the differentiations indicated in the eq. (3.1.5) we get:

$$
\begin{aligned}
& \frac{\hbar^{2}}{4 m^{2}}\left\{\frac{1}{\rho(x, t)} \frac{\partial^{3} \rho(x, t)}{\partial x^{3}}-\frac{2}{\rho^{2}(x, t)} \frac{\partial \rho(x, t)}{\partial x} \frac{\partial^{2} \rho(x, t)}{\partial x^{2}}+\right. \\
& \left.+\frac{1}{\rho^{3}(x, t)}\left[\frac{\partial \rho(x, t)}{\partial x}\right]^{3}\right\}=k(t) \times[x-q(t)] .
\end{aligned}
$$

To integrate the eq. (3.1.6) it is necessary to known the initial condition for $\rho(x, t)$. (Bassalo et al.,2002) Let us assume that for $t=0$ the physical system is represented by a normalized Gaussian wave packet, centered at $q(0)$, that is:

$\rho(x, 0)=\left[2 \pi \delta^{2}(0)\right]^{-1 / 2} \exp \left\{-\frac{[x-q(0)]^{2}}{2 \delta^{2}(0)}\right\}=\frac{1}{\sqrt{A}} \exp \left(-\frac{B^{2}}{C}\right)$

where:

$A=2 \pi \delta^{2}(0), \quad B=x-q(0), \quad C=2 \delta^{2}(0)$.

10)

Since the eq. (3.1.7) is a particular solution of the eq. (3.1.6), we must have:

$$
\begin{aligned}
& \frac{\hbar^{2}}{4 m^{2}}\left\{\frac{1}{\rho(x, 0)} \frac{\partial^{3} \rho(x, 0)}{\partial x^{3}}-\frac{2}{\rho^{2}(x, 0)} \frac{\partial \rho(x, 0)}{\partial x} \frac{\partial^{2} \rho(x, 0)}{\partial x^{2}}\right. \\
& + \\
& \left.+\quad \frac{1}{\rho^{3}(x, 0)}\left[\frac{\partial \rho(x, 0)}{\partial x}\right]^{3}\right\}=k(0) \times[x-q(0)] .
\end{aligned}
$$

Making the differentiation indicated in the eq. (3.1.11), results: (Bassalo et al.,2002)

\section{Error!}

Error! , (3.1.12)

and:

$$
\delta^{4}(0)=\frac{\hbar^{2}}{4 m^{2} k(0)}
$$

Comparing the eqs. $(3.1 .12,13)$ with the eqs. $(3.1 .6,7)$, by analogy we get:

Error! , (3.1.14)

$$
\rho(x, t)=\left[2 \pi \delta^{2}(t)\right]^{-1 / 2} \exp \left\{-\frac{[x-q(t)]^{2}}{2 \delta^{2}(t)}\right\},
$$

and: 
Error! . (3.1.16)

Using the eq. (2.1.3.13) we calculate the following differentiations (remembering that $t$ and $x$ as independent variables):

$\frac{\partial v_{q u}(x, t)}{\partial t}=\frac{\partial}{\partial t}\left\{\left[\frac{\dot{\delta}(t)}{\delta(t)}-\kappa\right] \times[x-q(t)]+\dot{q}(t)\right\}=$

$=\left[\frac{\ddot{\delta}(t)}{\delta(t)}-\frac{\dot{\delta}^{2}(t)}{\delta^{2}(t)}\right] \times[x-q(t)]-\left[\frac{\dot{\delta}(t)}{\delta(t)}-\kappa\right] \times \dot{q}(t)+\ddot{q}(t)$,

$(3.1 .17)$

$\frac{\partial v_{q u}(x, t)}{\partial x}=\frac{\partial}{\partial x}\left\{\left[\frac{\dot{\delta}(t)}{\delta(t)}-\kappa\right] \times[x-q(t)]+\dot{q}(t)\right\}=\frac{\dot{\delta}(t)}{\delta(t)}-\kappa$

Putting the eqs. $(2.1 .3 .13)$ and $(3.1 .17,18)$ into the eq. (3.1.4), considering the eq. (3.1.14), adding and subtracting the term $\omega^{2}(t) q(t)$, results: (Bassalo et al.,2002)

$$
\begin{aligned}
& {\left[\frac{\ddot{\delta}(t)}{\delta(t)}-\frac{\dot{\delta}^{2}(t)}{\delta^{2}(t)}\right] \times[x-q(t)]-\left[\frac{\dot{\delta}(t)}{\delta(t)}-\kappa\right] \times \dot{q}(t)+\ddot{q}(t)+} \\
& +\left\{\left[\frac{\dot{\delta}(t)}{\delta(t)}-\kappa\right] \times[x-q(t)]+\dot{q}(t)\right\} \times\left[\frac{\dot{\delta}(t)}{\delta(t)}-\kappa\right]+
\end{aligned}
$$

Error!

$\left[\frac{\ddot{\delta}(t)}{\delta(t)}-2 \frac{\dot{\delta}(t)}{\delta(t)} \kappa+\kappa^{2}+\omega^{2}(t)-\frac{\hbar^{2}}{4 m^{2} \delta^{4}(t)}\right] \times[x-q(t)]^{1}+$

$+\left[\ddot{q}(t)+\omega^{2}(t) q(t)\right] \times[x-q(t)]^{0}=0$.

To satisfy eq. (3.1.19), the following conditions must be obeyed:

$$
\frac{\ddot{\delta}(t)}{\delta(t)}-2 \frac{\dot{\delta}(t)}{\delta(t)} \kappa+\kappa^{2}+\omega^{2}(t)-\frac{\hbar^{2}}{4 m^{2} \delta^{4}(t)}=0 \rightarrow
$$$$
\ddot{\delta}(t)-2 \delta(t) \kappa+\left[\kappa^{2}+\omega^{2}(t)\right] \delta(t)=\frac{\hbar^{2}}{4 m^{2} \delta^{3}(t)},
$$

$$
\ddot{q}(t)+\omega^{2}(t) q(t)=0 .
$$

Putting:

Error! , (3.1.22)

we obtain: $\dot{\delta}(t)=\left(\frac{\hbar^{2}}{4 m^{2}}\right)^{1 / 4} \dot{\alpha}(t), \quad \ddot{\delta}(t)=\left(\frac{\hbar^{2}}{4 m^{2}}\right)^{1 / 4} \ddot{\alpha}(t)$.

$(3.1 .23,24)$

Inserting the eqs. (3.1.22-24) into the eq. (3.1.20) and multiplyng the result by $\alpha$, we get: (Bassalo et al., 2002)

$$
\ddot{\alpha}-2 \dot{\alpha} \kappa+\left(\omega^{2}+\kappa^{2}\right) \alpha=\frac{1}{\alpha^{3}} .
$$

Finally, eliminating the factor $\omega^{2}$ into the eqs. $(3.1 .20,21)$, we get:

$$
\begin{aligned}
& \ddot{\alpha}-2 \dot{\alpha} \kappa+\left(\omega^{2}+\kappa^{2}\right) \alpha=\frac{1}{\alpha^{3}} \rightarrow \\
& \ddot{\alpha} q-\ddot{q} \alpha-2 \dot{\alpha} \kappa q+\kappa^{2} \alpha q=\frac{q}{\alpha^{3}} \rightarrow
\end{aligned}
$$

$\frac{d}{d t}(\dot{\alpha} q-\dot{q} \alpha)=\frac{q}{\alpha^{3}}+2 \dot{\alpha} \kappa q-\kappa^{2} \alpha q \quad \rightarrow$

$(\dot{\alpha} q-\dot{q} \alpha) \frac{d}{d t}(\dot{\alpha} q-\dot{q} \alpha)=(\dot{\alpha} q-\dot{q} \alpha) \frac{q}{\alpha^{3}}+(\dot{\alpha} q-\dot{q} \alpha)\left(2 \alpha \kappa q-\kappa^{2} \alpha q\right)$

$\frac{d}{d t}\left[\frac{1}{2}(\dot{\alpha} q-\dot{q} \alpha)^{2}\right]+\frac{d}{d t}\left[\frac{1}{2}\left(\frac{q}{\alpha}\right)^{2}\right]=-\left(2 \dot{\alpha} \kappa q-\kappa^{2} \alpha q\right) \alpha^{2} \frac{d}{d t}\left(\frac{q}{\alpha}\right)$

$\underset{\rightarrow}{\frac{d}{d t}}\left\{\left[\frac{1}{2}(\dot{\alpha} q-\dot{q} \alpha)^{2}+\left(\frac{q}{\alpha}\right)^{2}\right]\right\}=-\left(2 \dot{\alpha} \kappa-\kappa^{2} \alpha\right) q \alpha^{2} \frac{d}{d t}\left(\frac{q}{\alpha}\right)$

$\frac{d I}{d t}=-\left(2 \dot{\alpha} \kappa-\kappa^{2} \alpha\right) q \alpha^{2} \frac{d}{d t}\left(\frac{q}{\alpha}\right)$,

where [see eq. (3.1)]:

$\mathrm{I}=\frac{1}{2}(\dot{\alpha} q-\dot{q} \alpha)^{2}+\left(\frac{q}{\alpha}\right)^{2}$

which represents the Ermakov-Lewis Invariant (ELI) of the TDHO. (Bassalo et al., 2002) In conclusion, the eq. (3.1.36) we have shown that the LNLS-NE has not an $E L /$ for the $T D H O$.

\section{The Bohmian Trajectories for the Logarithmic Nonlinear Schrödinger-Nassar Equation}

The associated Bohmian Trajectories (Nassar,2013), Sanz,2000),(Pan, 2010), (Holland, 2005), (Wyatt, 2005) for the Logarithmic Nonlinear Schrödinger-Nassar Equation (LNLS-NE) of an evolving ith particle of the 
42 Int. Res. J. Eng. Sci. Technol. Innov.

ensemble with an initial position $x_{0 i}$ can be calculated by considering that:

$$
\dot{x}_{i}(t)=v_{q u}\left[x_{i}(t), t\right] \text {. }
$$

Then substituting the eq. (4.1) into eq. (2.1.3.13), results:

$$
\begin{aligned}
& \dot{x}_{i}(t)=\left[\frac{\dot{\delta}(t)}{\delta(t)}-k\right] \times[x-q(t)]+\dot{q}(t) \rightarrow \\
& \dot{x}_{i}(t)-\dot{q}(t)=\left[\frac{\dot{\delta}(t)}{\delta(t)}-k\right] \times[x-q(t)] \\
& \frac{\dot{x}_{t}(t)-\dot{q}(t)}{x-q(t)}=\frac{\dot{\delta}(t)}{\delta(t)}-\kappa \rightarrow
\end{aligned}
$$

\section{Error!}

$$
\begin{aligned}
& \ln \frac{\left[x_{i}(t)-q(t)\right]}{\left[x_{0 i}-q_{0}\right]}=\ln \frac{\delta(t)}{\delta_{0}}-\kappa t \\
& \frac{\left[x_{i}(t)-q(t)\right]}{\left[x_{0 i}-q_{0}\right]}=\frac{\delta(t)}{\delta_{0}}-\exp (\kappa t) \rightarrow
\end{aligned}
$$

\section{Error! . (4.2)}

The eqs. $(3.1 .20,21)$ show that a continuous measurement of a quantum wave packet gives specific features to its evolution: the appearance of distinct classical and quantum elements, respectively. This measurement consists of monitoring the position of the quantum systems and the result is the measured classical path $q(t)$ for $t$ within a quantum uncertainty $\delta(t)$.

\section{The Bohmian Trajectories for the Logarithmic Nonlinear Schrödinger-Nassar Equation in Stationary Regime}

From the eqs. $(3 \cdot 1 \cdot 20,21)$, we note that for $k \neq 0$ a stationary regime can be reached and that the width of the wave packet can be related to the resolution of measurement as follows. Then, considering that $\delta(t)=c t e$ $[\dot{\delta}(t)=0]$ in the eqs. $(3.1 .20,21)$, we have:

$$
\begin{aligned}
& \text { Error!, } \\
& \text { where }^{[4]} \text { : } \\
& \tau_{B}=\left(\frac{2 m \delta_{0}^{2}}{\hbar}\right)=6,8 \times 10^{-26} s,
\end{aligned}
$$

is the Bohmtime constant which determines the time resolution of the quantum measurement, and:

$$
\dot{q}(t)+\omega_{0}^{2} q(t)=0 \rightarrow q(t)=q_{0} \exp \left( \pm i \omega_{0} t\right)
$$

The eqs. (4.3a,b) means that if an initially free wave packet is kept under a certain continuous measurement, its width $\left(\delta_{0}\right)$ may not spread in time. Then, the associated Bohmnian Trajectories (BT) [eq. (4.2)] of an evolving ith particle of the ensemble with an initial position $x_{0 i}$ is giving by:

$$
x_{i}(t)=q_{0} \exp \left( \pm i \omega_{0} t\right)+\left(x_{0 i}-q_{0}\right) \exp (-\kappa t)
$$

\section{REFERENCES}

Feynman RP (1948). Reviews of Modern Physics 20: 367.

Feynman RP, Hibbs AR (1965). Quantum Mechanics and Path Integrals, McGraw-Hill Book Company, USA.

Bernstein IB (1985). Physical Review A32: 1.

Nassar AB (2013). Annals of Physics 331: 317.

Madelung E (1926). Zeitschrift für Physik 40: 322.

Bohm D (1952). Physical Review 85: 166.

Bassalo JMF, Alencar PTS, Cattani MSD, Nassar AB (2002).Tópicos da Mecânica Quântica de de Broglie-Bohm, EDUFPA; -----. 2010. EBook (http://publica-sbi.if.usp.br/PDFs/pd1655.pdf.

See books on the Fluid Mechanics, for instance:

Streeter VL, Debler WR. (1966). Fluid Mechanics, McGraw-Hill Book Company, Incorporation.

Coimbra AL (1967). Mecânica dos Meios Contínuos, Ao Livro Técnico S. A.

Landau L, Lifshitz E (1969). Mécanique des Fluides. Éditions Mir.

Bassalo JMF (1973). Introdução à Mecânica dos Meios Contínuos, EDUFPA.

Cattani MSD (1990/2005). Elementos de Mecânica dos Fluidos, Edgard Blücher.

Bassalo JMF, Alencar PTS, Silva DG, Nassar AB, and Cattani MSD (2010). arXiv:1010.2640v1. [quant-ph].

Nassar AB (1986). Journal of Mathematical Physics 27: 2949.

Lewis HR. (1967). Physical Review Letters, 18(13): 510; 18(15): 636(E). Ermakov VP (1880). Universitetskie Izvestiya, Kiev, 9: 1.

Nassar AB (1986a). Journal of Mathematical Physics, 27(3): 755; (1986b). Journal of Mathematical Physics, 27(12): 2949;-(1986c). Physical Review A 33(3): 2134.

Sanz AS, Borondo F, Miret-Artés S (2000). Physical Review B 61: 7743. Pan AK (2010). Pram. Journal of Physics 74: 867.

Holland P (2005). Ann. Phys. 315: 505.

Wyatt RE (2005). Quantum Dynamics with Trajectories, Springer, New York.

How to cite this article: Bassalo J.M.F., Nassar A.B. and Cattani M.S.D. (2014). Feynman propagator, ermakov-lewis invariant and bohmian trajectories for the logarithmic nonlinear schrödinger-nassar equation. Int. Res. J. Eng. Sci. Technol. Innov. 3(3):35-42 